\title{
Millimeter Wave Radar Imaging for Non-Destructive Detection of Material Defects
}

\author{
Ingrid Ullmann ${ }^{1}$, Julian Adametz ${ }^{1}$, Daniel Oppelt ${ }^{1}$, Martin Vossiek ${ }^{1}$ \\ ${ }^{1}$ Institute of Microwaves and Photonics, Friedrich-Alexander-University Erlangen-Nuremberg, \\ Cauerstr. 9, 91058 Erlangen, Germany \\ ingrid.ullmann@fau.de
}

\begin{abstract}
This contribution presents a novel method for the detection of material defects by means of millimeter wave radar imaging. Unlike common radar signal processing algorithms, which are usually based on the assumption of free space propagation of the electromagnetic wave, our approach also considers the effects occurring at material discontinuities, namely refraction and the change in phase velocity. Therefore, by means of the developed reconstruction algorithm, we are able to correctly depict the inside of objects made of refractive materials, such as most polymers. The method was experimentally verified by reconstructing a polyethylene object with air inclusions. Here, the proposed algorithm was able to depict the scenario very well.
\end{abstract}

Key words: sub-surface imaging, non-destructive testing, millimeter wave SAR, reconstruction algorithm, refraction.

\section{Introduction}

Throughout the whole process chain, quality management is a fundamental task in industrial production. The monitoring of devices and materials is a core issue in order to guarantee a consistent quality of the products. Here, often the interior of devices is of essential interest, for example in the detection of material defects.

When the objects under test are not transparent for the human eye, wave based imaging, employing electromagnetic or acoustic waves, can be applied. There is a variety of wave based techniques utilized for non-destructive material testing. Among them are microwave and terahertz radar, ultrasound, X-ray tomography and many more.

In this contribution we present an imaging method based on synthetic aperture radar (SAR). The SAR technique originates from remote sensing - therefore SAR imaging algorithms usually are based on the assumption of a free space propagation of the electromagnetic (EM) wave. This assumption still holds when screening dielectric devices which exhibit a relative electrical permittivity equal (or very close) to one. However, when applying such algorithms to a scenario in which the wave propagates through materials of a refractive index significantly greater than one, the reconstruction is based on false assumptions. Consequently, the reconstructed image will be of low quality, it may even be faulty. The reasons for this misrepresentation are the refraction of the wave and the change in phase velocity occurring at the material boundary. These effects are illustrated in Fig. 1. On the left the object displays the same material behavior as the free space surrounding it, on the right it does not.
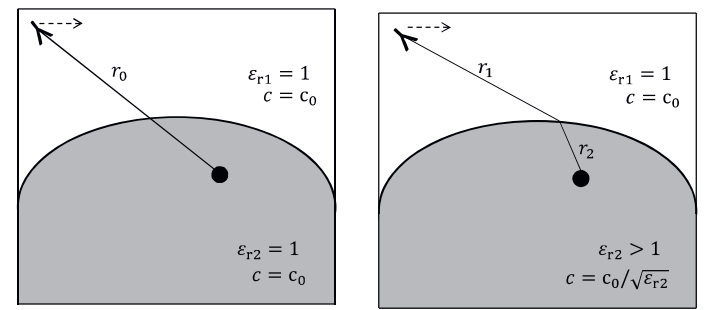

Fig. 1. Reflective SAR measurements depending on the permittivity of the material under test (gray)

This paper presents a method to overcome the problem. We developed a new reconstruction approach which takes into consideration the effects named above. It can therefore be applied not only to surroundings that exhibit a freespace-like behavior, but also to the sub-surface imaging of refractive materials, which includes many polymers, too. 
Radar signal processing algorithms for subsurface imaging can be found in the literature:

One approach is to reconstruct an image by numerically estimating the permittivity distribution in the object under test [1], [2]. This method however is very demanding in terms of computational capacities.

Other imaging methods originate from typical radar applications such as security screening [3], [4] and ground-penetrating radar (GPR). While in security screening usually the clothing of a person is transparent for radar waves, GPR represents a very similar scenario to the one described.

Often though, GPR algorithms assume that the transceiving antennas are situated directly above the ground. Then refraction does not have to be considered. Approaches taking into account a certain height of the antenna, and, consequently, refraction, can be found in the literature, too [5]-[7]. However GPR usually assumes a plane material boundary. The algorithm proposed in this contribution examines the more general case of an arbitrarily shaped surface.

\section{Theory}

The propagation of an electromagnetic wave can be described by the Helmholtz equation for the electric field

$$
\nabla^{2} \vec{E}(t)-c \frac{\partial^{2}}{\partial t} \vec{E}(t)=0
$$

and analogously for the magnetic field. (1) is a homogeneous wave equation, with $c$ denoting the wave's propagation velocity. One possible solution of the partial differential equation (1) is a plane wave. Assuming the wave to propagate in a direction $x$, then the plane wave equation reads

$$
E(x, t)=|E| \cdot \exp [j(\omega t-k x)]
$$

Here, $k$ is the wavenumber, related to the wavelength $\lambda$ by

$$
k=\frac{2 \pi}{\lambda} .
$$

The phase velocity $c$ is

$$
c=\frac{\omega}{k}=\frac{1}{\sqrt{\mu \varepsilon}}=\frac{1}{\sqrt{\mu_{0} \mu_{\mathrm{r}} \varepsilon_{0} \varepsilon_{\mathrm{r}}}},
$$

depending on the angular frequency $\omega$ and the material's parameters $\mu_{r}$ and $\varepsilon_{r}$ (relative magnetic permeability and relative electric permittivity, respectively). When considering only non-magnetic materials as is done here, the phase velocity in a material is

$$
c=\frac{c_{0}}{\sqrt{\varepsilon_{\mathrm{r}}}}=\frac{c_{0}}{n}
$$

where $c_{0}$ is the speed of light and $n$ is the refractive index of the material.

When passing through a boundary between two materials of different refractive indices, an EM wave will be refracted according to Snell's law

$$
\frac{\sin \alpha}{\sin \beta}=\frac{n_{2}}{n_{1}}
$$

where $\alpha$ denotes the angle of incidence and $\beta$ the angle of refraction. Snell's law is a consequence of Fermat's principle - (6) minimizes the wave's travel time when a discontinuity of material is present [8].

\section{Modeling of the Scenario}

We investigate two scenarios: a planar and a non-planar material boundary. The respective models are shown in Figs. 2 and 3. Here, the indices $A, T$, and $B$ denote the coordinates of the antenna, the boundary and the target, respectively.

For the sake of simplicity, all considerations are derived for a 2D setup. An extension to the third dimension will be shown below.

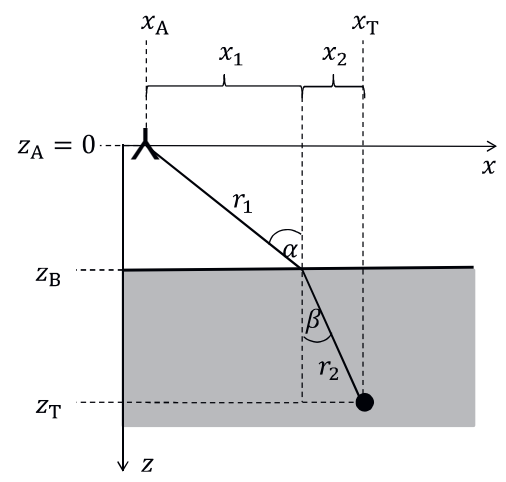

Fig. 2. Model with planar material boundary

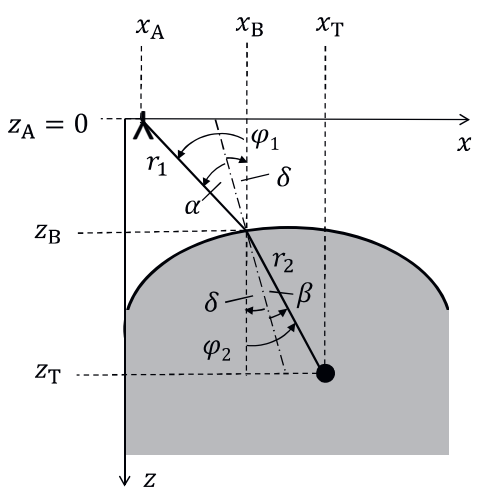

Fig. 3 Model with non-planar material boundary 
The coordinate system employed can be seen from the figures: The lateral direction is $x$ (and $y$ in 3D), the radial (range) direction is $z$.

The reconstruction domain is divided into two sub-volumes. The first one is modeled to be free space, i.e. its relative permittivity is equal to one. Surrounded by the free space is the device under test (DUT) of material two. Material two's permittivity is assumed to be a known value greater than one. It is modeled to be frequencyindependent and lossless (i.e. real-valued). Both materials are nonmagnetic, i.e. they display the permeability of vacuum $\mu_{0}$. The DUT can be arbitrarily shaped, but the shape is assumed to be known.

We use a monostatic synthetic aperture radar, transmitting a signal $s_{\mathrm{Tx}}(t, f)$. The receiver signal $s_{\mathrm{Rx}}(t, f)$ is the wave backscattered from the DUT. The antenna positions spanning the synthetic aperture are equidistantly spaced and they are located at $z=0$. The antenna itself is modeled as an isotropic radiator. It is presumed that the volume to be reconstructed is located in the antenna's far-field. Therefore, the incident waves can be considered to be plane waves.

According to the antenna's isotropic directivity pattern, some part of the radiated electromagnetic field will be radiated in such a way that after traversing medium one and being refracted at the boundary it will actually meet the target ( $r_{1}$ and $r_{2}$ in Figs. 2 and 3 ). Assuming isotropic scattering, some part of the reflected field will traverse the same way back to the antenna. Then, the received signal's phase will be

$$
\varphi_{\mathrm{Rx}}=\omega \cdot 2 \cdot \frac{r_{1}+r_{2} \sqrt{\varepsilon_{\mathrm{r} 2}}}{c_{0}},
$$

which follows from (2) - (4).

In (7), the phase offset due to the scatterer's reflection properties will be neglected for it is a constant and thus will not influence the reconstruction process.

Furthermore, since the reconstruction method will only evaluate the phase, the signal's amplitude does not need to be considered. Accordingly, the received backscatter signal becomes

$$
s_{\mathrm{Rx}}=1 \cdot \exp \left(-j \varphi_{\mathrm{Rx}}\right)
$$

when setting the amplitude to a virtual value of one and inserting (7) for the phase.

\footnotetext{
${ }^{1}$ An incident angle of $90^{\circ}$ is excluded because of the tangent function in (9)
}

\section{Image Reconstruction Approach}

The image reconstruction is based on the spatial matched filter approach. Here, for each pixel (or voxel in 3D) a signal hypothesis is stated. This hypothesis equals the signal which the antenna would receive if there was a point scatterer located in the respective place. In a next step, the hypotheses are correlated to the actually measured signal for all antenna positions. Those points in which a scattering truly occurs will then exhibit a high value for the correlation. All others will exhibit a low value. By converting the magnitude of the correlation to a brightness scaling, the image is generated.

It is obvious that in order to state the hypotheses, the path traversed by the wave needs to be known. This is trivial in a free space surrounding: then, it equals the direct way between the antenna and the respective point. In the heterogeneous case however, the wave will be refracted according to (6). However, the two angles $\alpha$ and $\beta$ are not known a priori due to the isotropic radiation pattern.

Therefore, the algorithm first has to determine the wave's path and then can proceed to the correlation procedure described above.

In the following, some possibilities to find the optical path are described for the two scenarios of a planar and a non-planar boundary.

\section{Planar Material Boundary}

From Fig. 2 it can be seen that

$$
\begin{gathered}
x_{\mathrm{T}}=x_{\mathrm{A}}+x_{1}+x_{2}= \\
x_{\mathrm{A}}+\tan (\alpha) \cdot z_{\mathrm{B}}+\tan (\beta) \cdot\left(z_{\mathrm{T}}-z_{\mathrm{B}}\right)
\end{gathered}
$$

where $\beta$ can be replaced using (6). Therefore, (9) can be formulated depending on $\alpha$ only. However, it cannot be solved for $\alpha$ directly. In order to find the true incident angle, a sweep for $\alpha=]-90^{\circ}, 90^{\circ}\left[\right.$ can be conducted $^{1}$, searching for that value of $\alpha$ that will best satisfy (9). When it is found, the value for $\beta$ can be deduced from Snell's law and the signal hypotheses can be formulated according to (7) and (8). The lengths $r_{1}$ and $r_{2}$ can be derived from geometric relations:

and

$$
r_{1}=\frac{z_{\mathrm{B}}}{\cos (\alpha)}
$$

$$
r_{2}=\frac{z_{\mathrm{T}}-z_{\mathrm{B}}}{\cos (\beta)} \text {. }
$$




\section{Non-Planar Material Boundary}

In the general case the approach described above can no longer be utilized: For one thing, now the $z$-coordinate of the transit $z_{\mathrm{B}}$ is no longer constant. Secondly, the angles $\alpha$ and $\beta$ are defined with respect to the normal vector in their transit point - which in the case of an arbitrary surface no longer coincides with the vertical.

As a solution, in that case we did not vary the incident angle. Instead we searched for that point within the boundary which is the true point of transit between the two media. From it, the optical path can be derived directly.

From the geometry in Fig. 3 it can be seen that the incident angle is

$$
\alpha=\tan ^{-1}\left(\frac{x_{\mathrm{B}}-x_{\mathrm{A}}}{z_{\mathrm{B}}}\right)+\delta .
$$

Herein, $\delta$ is the angular difference between the vertical and the normal in the respective point $\left(x_{\mathrm{B}} \mid z_{\mathrm{B}}\right)$.

Likewise, for $\beta$ we can write

$$
\beta=\tan ^{-1}\left(\frac{x_{\mathrm{T}}-x_{\mathrm{B}}}{z_{\mathrm{T}}-z_{\mathrm{B}}}\right)+\delta .
$$

Additionally, $\alpha$ and $\beta$ also have to fulfill the law of refraction (6). That point of the boundary $\left(x_{\mathrm{B}} \mid z_{\mathrm{B}}\right)$ which fits (6), (12) and (13), is the true point of refraction. From it, the lengths $r_{1}$ and $r_{2}$ are easily found by the Pythagorean theorem:

and

$$
r_{1}=\sqrt{\left(x_{\mathrm{B}}-x_{\mathrm{A}}\right)^{2}+z_{\mathrm{B}}^{2}}
$$

$$
r_{2}=\sqrt{\left(x_{\mathrm{T}}-x_{\mathrm{B}}\right)^{2}+\left(z_{\mathrm{T}}-z_{\mathrm{B}}\right)^{2}} .
$$

Again, having determined $r_{1}$ and $r_{2}$, the signal hypothesis follows from (7) and (8).

\section{Experimental Verification}

Measurements were conducted to demonstrate the algorithm's feasibility. Here, we examined a polyethylene (PE) object into which two holes were drilled. The drill holes represent air inclusions within the material. Such imperfections can be caused in the production process of the polymer or in the operation of a component.

The object under test is depicted in Fig. 4. It was constructed to be invariant along the vertical direction, thus allowing for a two-dimensional scanning and reconstruction. Its surface was chosen to be non-planar and non-symmetric in order to demonstrate the algorithm's capacity to reconstruct rather complex objects.
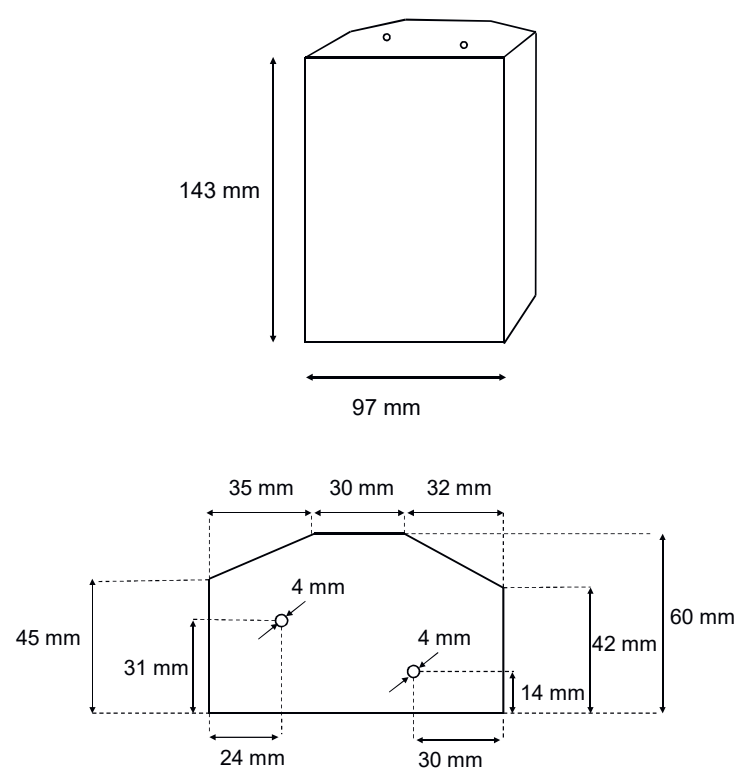

Fig. 4 Sketch of the object under test and its crosssection with all relevant measures

We employed a quasi-monostatic setup (i.e. transmitter and receiver in close proximity), consisting of two H-polarized horn antennas. The transmitted signal, generated by a network analyzer with frequency extenders, was a 201point SFCW (stepped frequency continuous wave) signal in the W-band (75 to $110 \mathrm{GHz}$ ). The corresponding free space wavelengths cover a range from $2.7 \mathrm{~mm}$ to $4 \mathrm{~mm}$. The radiated power was $-17 \mathrm{dBm}(20 \mu \mathrm{W})$.

In order to generate a 2D image, a line aperture is sufficient. From the spatial sampling theorem, the spacing between the antenna array positions must not exceed

$$
\Delta d_{\max }=\lambda_{\min } / 2
$$

[9], which is $1.35 \mathrm{~mm}$ for the W-band. Here, a spacing of $1 \mathrm{~mm}$ was chosen.

The results obtained are shown in Fig. 5 . Depicted from top to bottom are a sketch of the cross section of the sample, an image obtained from a classical free space reconstruction and the image reconstructed by the developed algorithm. Herein, we considered the polyethylene to display a relative permittivity of 2.3 in the relevant frequency range [10].

The object's contour is illustrated by the dashed lines. For the sake of simplicity, in Fig. 5, the object is depicted in a local coordinate system, starting from $z=0$ at the DUT's front boundary. In the global coordinate system, the object was 
located at a range of $25.8 \mathrm{~cm}$ from the aperture plane.
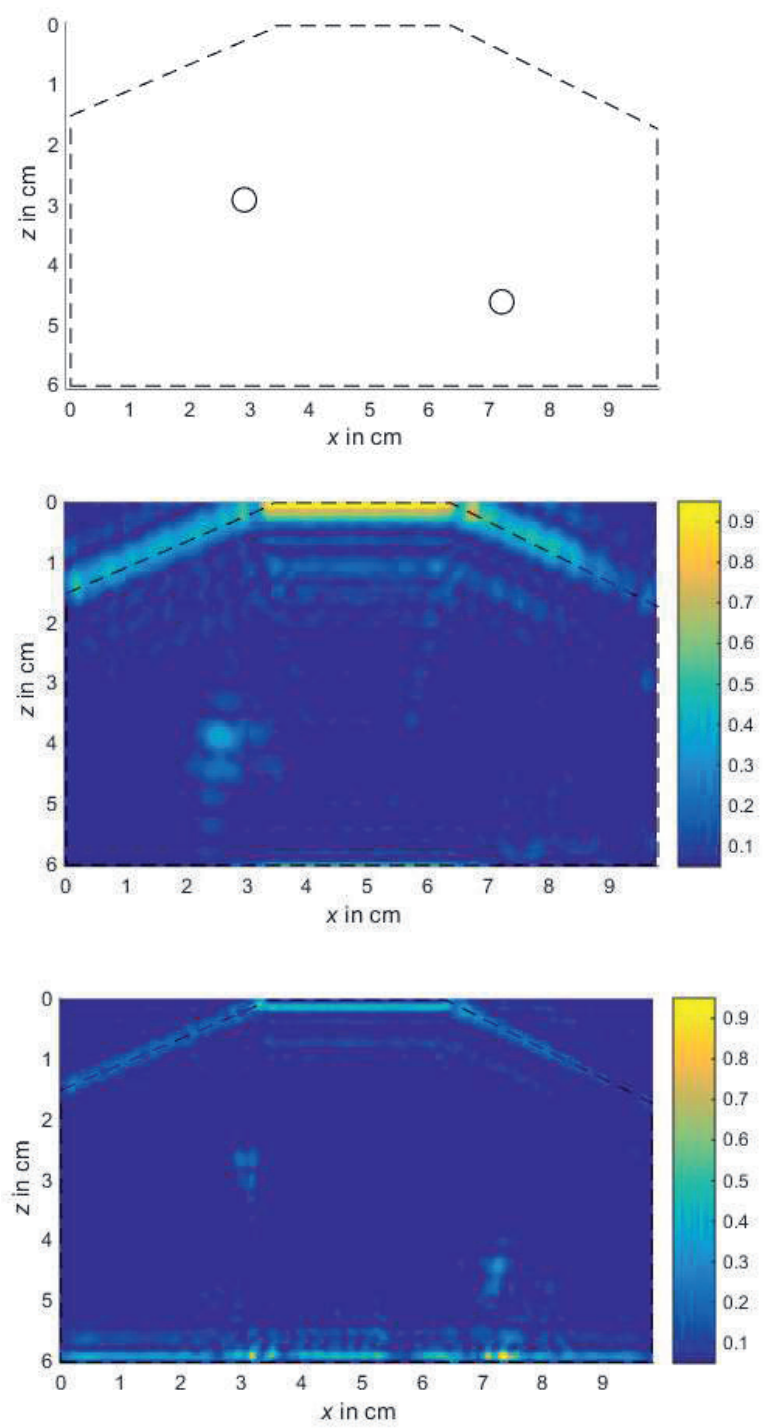

Fig. 5 Reconstruction of the polyethylene object under test. From top to bottom: Geometry sketch, free space reconstruction, and adapted reconstruction

Note that only the targets' upper and lower boundaries are reconstructed. They correspond to the material discontinuities, at which reflections occur.

From Fig. 5 it can be seen that the adapted procedure depicts the scenario very well. Both targets are reconstructed at their true positions. By contrast, the free space reconstruction, does not depict the target at $z \approx 4.5 \mathrm{~cm}$ within the object. The lower boundary is not visible either.

The improvement in the localization is because in the free space case the propagation velocity is assumed too high. Consequently, since the velocity is proportional to the traversed way, a too long distance along the range direction is reconstructed. With the adapted algorithm this error is not made.

Furthermore, with the developed method the targets are focused more precisely. Since the lateral resolution depends on the wavelength and the radial one depends on the phase velocity, a better resolution can be obtained when taking the material characteristics into account [4]. This effect is also visible at the material boundary.

\section{Extension to the Third Dimension}

Both the procedures described above can be extended to the third dimension.

From Fermat's principle it follows that an incident ray and its refraction will be the same plane [11]. Consequently, in the 3D case, a generalized lateral coordinate $l$ is introduced, which is defined by

$$
l=\sqrt{x^{2}+y^{2}} .
$$

This generalized coordinate accounts for the propagation plane. Then, the procedures described above can be adapted analogously, replacing the coordinate $x$ by $l$.

\section{Conclusion}

We extended a SAR-based imaging principle to the handling of two-media systems, consisting of free space and a refractive material. It is suitable for sub-surface imaging of devices made of dielectric, refractive materials such as polymers. As an example, we showed the detection of material defects, namely air inclusions, in a polyethylene object. Here we compared the developed method to a conventional free space reconstruction. The adapted algorithm yielded better results than the conventional one with respect to the localization of the targets as well as with respect to the focusing.

The proposed method is suitable for two- and three-dimensional image reconstructions. It was derived for monostatic SAR but can be extended to a multistatic setup in the same way.

Future research will focus on the automated detection of the DUT's characteristics, such as its contour and material properties.

\section{Acknowledgment}

The authors would like to thank the European Regional Development Fund (ERDF) for funding parts of the research activities. This work is part of the FAU project "Advanced Analytics for Production Optimization" (E|ASY-Opt) funded by the Bavarian program "Investment for growth and jobs" 2014-2020 (Bavarian Ministry of Economic Affairs and Media, Energy and Technology). 


\section{References}

[1] J. De Zaeytijd, A. Franchois, C. Eyraud, and J.-M. Geffrin, Full-Wave Three-Dimensional Microwave Imaging With a Regularized GaussNewton Method - Theory and Experiment, IEEE Transactions on Antennas and Propagation. 55, 3279-3292 (2007); doi: 0.1109/TAP.2007.908824

[2] L. Garnero, A. Franchois, J.-P. Hugonin, C. Pichot, and N. Joachimowitz, Microwave Imaging - Complex Permittivity Reconstruction by Simulated Annealing, IEEE Transactions on Microwave Theory and Techniques 39, 18011807 (1991); doi: 10.1109/22.97480

[3] S. Ahmed, O. Ostwald, and L.-P. Schmidt, Automatic Detection of Concealed Dielectric Objects for Personnel Imaging, IEEE MTT-S International Microwave Workshop Wireless Sensing, Local Positioning, RFID, Cavtat, Croatia, 1-4 (2009)

[4] S. S. Ahmed, A. Schiessl, F. Gumbmann, M. Tiebout, S. Methfessel, and L.-P. Schmidt Advanced Microwave Imaging, IEEE Microwave Magazine 13, 26-43 (2012); doi: 10.1109/ MMM.2012.2205772

[5] C. M. Rappaport, Accurate Determination of Underground GPR Wavefront and B-Scan Shape From Above-Ground Point Sources", IEEE Transactions on Geoscience and Remote Sensing 45, 2429 - 2434 (2007); doi: 10.1109/TGRS.2007.901004

[6] C. Rappaport, A Novel, Non-Iterative Method to Find the Surface Refraction Point for Air-Coupled Ground Penetrating Radar, Proc. 5th EuCAP, Rome, Italy, 1786-1789 (2011)

[7] A. Heinzel, M. Peichl, E. Schreiber, F. Bischeltsrieder; S. Dill, S. Anger, T. Kempf, and M. Jirousek, Focusing Method for Ground Penetrating MIMO SAR Imaging within HalfSpaces of Different Permittivity, Proc. 11th EUSAR, Hamburg, Germany, 842-846 (2016)

[8] E. Hecht, Optics, 5th ed., Pearson, London, Great Britain, 2002

[9] J. Benetsy, J. Chen, and Y. Huang, Microphone Array Signal Processing, Springer, Berlin, Germany, 2008; doi: 10.1007/978-3-540-78612-2

[10] Ullmann's Polymers and Plastics, Wiley-VCH, Weinheim, Germany, 2016

[11] M. Born, E. Wolf, Principles of Optics, 6th ed., Pergamon Press, Oxford, Great Britain, 1980 